\title{
Investigation on Hemodialysis Membranes Morphology and Human Serum Proteins Depositions Using Synchrotron-based Imaging
}

\author{
Amira Abdelrasoul ${ }^{1,2}$ \\ ${ }^{1}$ Department of Chemical and Biological Engineering, University of Saskatchewan, 57 Campus Drive, Saskatoon, \\ Saskatchewan, S7N 5A9, Canada. \\ ${ }^{2}$ Division of Biomedical Engineering, University of Saskatchewan, 57 Campus Drive, Saskatoon, Saskatchewan, S7N 5A9, \\ Canada. \\ amira.abdelrasoul@usask.ca
}

\section{Extended Abstract}

End stage renal disease (ESRD) affects $\sim 10 \%$ of the world's population. Hemodialysis (HD) is a life-sustaining extracorporeal blood purifying method for patients with ESRD or kidney failure. This membrane-based therapy is associated with acute side effects, life-threatening chronic conditions, and unacceptably high morbidity and mortality rates. Due to the lack of endothelium functionality of the polymeric surfaces of HD membranes, their interaction with human serum proteins induces an inflammatory response and leads to numerous long-term clinical consequences that are in part determined by the degree of membrane biocompatibility[1]. Blood-membrane interactions trigger a multifaceted series of events of protein adsorption, in addition to the activation blood cells such as leukocytes, platelets, and red blood cells (RBCs) or indirectly activate them through a pathway that activates the complement system or coagulation factors [2,3]. Therefore, protein adsorption provokes a series of biochemical reactions that can lead to serious long- and short-term side effects in HD patients [4]. In addition, the attachment of human serum proteins on membrane surface would significantly reduce uremic toxins clearance efficiency, especially protein bound uremic toxins and middle molecules. Membrane characteristics such as chemical composition, hydrophilicity, surface roughness, pore size and binding affinity influence the interactions and the attachments between human serum proteins and HD membranes hence they can impact the inflammatory response experienced by HD patients. This study highlighted an innovative investigation on the common HD membrane morphology available in Canadian hospitals, and its affinity for human serum protein attachment. Synchrotron based X-ray imaging techniques available at the Biomedical Imaging and Therapy (BMIT) beamline at the Canadian Light Source (CLS) has answered key questions of HD membrane morphology layer-by-layer and human serum protein transport in membrane channels. This study will highlight the proof that Synchrotron imaging techniques are effective for membrane characterization and protein deposition assessment through both qualitative and quantitative analyses.

\section{References}

[1] P. Faria, M. Costa, C. F. Franssen, W. J. Van Son, S. P. Berger, “ The complement system in dialysis: a forgotten story, "Front. Immunol., vol. 9, pp.71, 2018.

[2] A. K. Mohiuddin, "Patient safety: a nobody's concern," Int. J. Hosp. Pharm., vol. 4, pp. 22, 2019.

[3] M. He, X. Cui , H. Jiang, X. Huang, W. Zhao, C. Zhao, "Super-anticoagulant heparin-mimicking hydrogel thin film attached substrate surfaces to improve hemocompatibility," Macromol. Biosci., vol 17, pp. 1600281.

[4] A. Mollahosseini, A. Abdelrasoul, A. Shoker, A., "A critical review of recent advances in hemodialysis membranes hemocompatibility and guidelines for future development," Mater. Chem. Phys., vol 248, pp.122911, 2020. 